\title{
Seismic response of linear accelerators
}

\author{
C. Collette, K. Artoos, M. Guinchard, and C. Hauviller \\ CERN, CH-1211 Geneva 23, Switzerland \\ (Received 20 November 2009; published 26 July 2010)
}

\begin{abstract}
This paper is divided into two parts. The first part presents recent measurements of ground motion in the LHC tunnel at CERN. From these measurements, an update of the ground motion model currently used in accelerator simulations is presented. It contains new features like a model of the lateral motion and the technical noise. In the second part, it is shown how this model can be used to evaluate the seismic response of a linear accelerator in the frequency domain. Then, the approach is validated numerically on a regular lattice, taking the dynamic behavior of the machine alignment stage and the mechanical stabilization of the quadrupoles into account.
\end{abstract}

DOI: 10.1103/PhysRevSTAB.13.072801

PACS numbers: 07.10.-h

\section{INTRODUCTION}

The basic principle of future compact linear colliders is to accelerate two beams of elementary particles (electrons, positrons) before colliding them at an interaction point. These machines are expected to be extremely long, and be composed of a series of accelerating structures and electromagnets (of various sizes) with fields focusing the beams. The number and quality of the collisions relies on the capability of the machine to control the size and direction of the beams with extreme precision, not only at the interaction point, but also in the whole machine. To this purpose, each of the electromagnets should be extremely stable and capable to point in the right direction. Actually, the main source of excitation is the ground vibrations transmitted to the quadrupoles [1-3]. They have basically two origins: earth activity and cultural noise. The first one is a superposition of waves arising, among others, from valley resonances and ocean resonances, known as microseismic waves. It results in a coherent motion of the ground in the very low frequency range [below $\sim 2(\mathrm{~Hz})]$. The second contribution results from the human activities, e.g., cooling system or ventilation. It is dominant above a few Hertz, and usually not coherent. Actually, the coherence between different points in the tunnel is an essential characteristic for linear colliders, because a fully coherent motion of the whole line would not affect its performances. More precisely, let us consider a line of quadrupoles represented in Fig. 1 (sometimes also called lattice). $L$ denotes the length of the line and $d$ the distance between quadrupoles.

Depending on the wavelength of the ground waves $\lambda$, the relative motion between quadrupoles can be divided into three categories, as shown in Table I.

Therefore, the better the knowledge of the ground vibration, the more precise will be the prediction of the accelerator performances and the more adapted will be the counteractions to the seismic excitations.

The motivation of this paper is twofold: first, to update the ground motion model of the LHC tunnel, and include new features like local excitations and a model of the ground motion in the lateral direction; second, to show how the model can be used to study the seismic response of a linear accelerator, and evaluate the beam vibration when the support dynamics is taken into account.

The paper is organized as follows. Section II reviews the basic mathematical quantities used for the characterization of the ground motion. Section III presents the measurements of the ground motion performed in the LHC tunnel. Section IV presents the new model of the ground motion calibrated on these measurements. Section V explains how to use the model to evaluate the seismic response of a linear accelerator and presents a numerical validation of the model on a regular focusing lens-open space-defocusing lens-open space (FODO) lattice. Finally, Sec. VI draws the conclusions.

\section{GROUND VIBRATIONS BACKGROUND}

\section{A. Absolute motion}

Let $x(t)$ be the time history of the vertical motion of the ground at one point. The periodicity of the signal can be estimated by measuring its autocorrelation function [4]

$$
R_{x x}(\tau)=\int_{-\infty}^{\infty} x(t) x(t+\tau) d t
$$

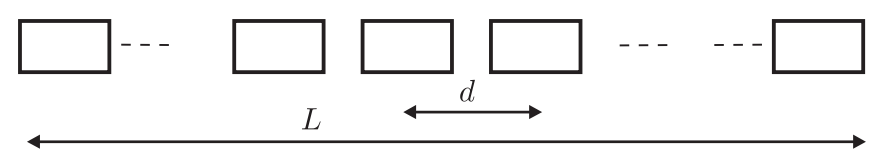

FIG. 1. Lattice of quadrupoles. 
TABLE I. Classification of the quadrupole relative motion.

\begin{tabular}{lc}
\hline \hline$\lambda<d$ & Corresponds to high frequencies. \\
& Quadrupoles are not correlated. \\
$d<\lambda<L$ & Quadrupoles are correlated \\
$L<\lambda$ & Global displacement of the lattice, \\
& does not affect its performance. \\
\hline \hline
\end{tabular}

The Fourier transform of $R_{x x}(\tau)$ is called the power spectral density (PSD) $\Phi_{x x}(\omega)$ (or simply $\Phi_{x}$ ) of $x(t)$

$$
\Phi_{x x}(\omega)=\int_{-\infty}^{\infty} R_{x x}(\tau) e^{-i \omega \tau} d \tau
$$

The root mean square (RMS) value is given by

$$
\sigma_{x}=\sqrt{\int_{-\infty}^{+\infty} \Phi_{x x}(\omega) d \omega}
$$

which is infinite because the ground motion is not a stationary signal. However, one can still calculate the contribution to $\sigma_{x}$ above a given angular frequency $\omega$ by

$$
\sigma_{x}(\omega)=\sqrt{\int_{\omega}^{\infty} \Phi_{x x}(\nu) d \nu}
$$

As explained in the Introduction, the correlation between the motion of different points is also an important quantity for the evaluation of the performances of linear accelerators. Let $x(t)$ and $y(t)$ be the vertical motion of two points separated by a distance $L$. One can define their cross-spectral density or mutual spectral density by

$$
\Phi_{x y}(\omega)=\int_{-\infty}^{\infty} R_{x y}(\tau) e^{-i \omega \tau} d \tau
$$

and the normalized power spectral density is defined by

$$
\gamma_{x y}(\omega)=\frac{\Phi_{x y}(\omega)}{\sqrt{\Phi_{x x}(\omega) \Phi_{y y}(\omega)}} .
$$

The real part of (6) is called the correlation and its module is called the coherence.

\section{B. Relative motion}

For the alignment of a linear accelerator, it is also interesting to know the relative motion of the ground between different locations in the tunnel. Let $d(t)=$ $x_{1}(t)-x_{2}(t)$ be the relative motion between two points located at a distance $L$, the power spectrum of the $d(t)$ is given by

$$
\begin{aligned}
\rho\left(\omega, x_{1}, x_{2}\right) & =\lim _{T \rightarrow \infty} \frac{1}{T}\left|\int_{-T / 2}^{T / 2} d(t) e^{-i \omega t} d t\right|^{2} \\
& =\int_{-\infty}^{\infty} R_{d d}(\tau) e^{-i \omega \tau} d \tau .
\end{aligned}
$$

Typically, at low frequencies, the amplitude of this spectrum is smaller than $\Phi_{x_{1} x_{1}}(\omega)$ or $\Phi_{x_{2} x_{2}}(\omega)$ : it grows in $\omega^{-2}$ instead of $\omega^{-4}$ [5]. At higher frequencies (typically higher than a few Hertz) the correlations disappear and the spectra become similar. It is possible to show [6] that $\rho(\omega, L)$ is related to the usual spectrum by

$$
\begin{aligned}
\rho\left(\omega, x_{1}, x_{2}\right)= & \Phi_{x_{1} x_{1}}(\omega)+\Phi_{x_{2} x_{2}}(\omega)-\Phi_{x_{1} x_{2}}(\omega) \\
& -\Phi_{x_{2} x_{1}}(\omega) .
\end{aligned}
$$

Assuming that the spectra of the signals are the same $\Phi_{x_{1} x_{1}}(\omega)=\Phi_{x_{2} x_{2}}(\omega)=\Phi_{x x}(\omega)$, Eq. (8) becomes

$$
\rho\left(\omega, x_{1}, x_{2}\right)=\Phi_{x x}(\omega) 2\left\{1-\operatorname{Re}\left[\gamma_{x_{1} x_{2}}(\omega)\right]\right\} .
$$

Note that if the excitation field is spatially homogenous, the relative spectral density depends only upon the difference $x_{1}-x_{2}$ and $\rho\left(\omega, x_{1}, x_{2}\right)=\rho(\omega, L)$.

Pioneering activities in the description of ground motion have been reported in $[7,8]$, and later in the accelerator community [9-12]. In order to separate the contributions of the different spatial wavelengths to the relative motion between the two points, a two-dimensional power spectrum has been introduced in $[5,13]$. The motivation to introduce such a quantity is the following one. On the one hand, if the ground motion displaces the linear accelerator as a whole rigid body, it would not influence its operation. This is, for example, the case for long wavelengths resulting from the ocean resonances. Even if they have large amplitudes, these waves have a low influence because of their long wavelength. On the other hand, vibrations with a spatial wavelength of a few tens of meters can have a great effect, even though their amplitudes are much smaller.

If $s$ is the longitudinal coordinate along the accelerator, the two-dimensional power spectrum of $x(t, s)$ is defined in [13] as

$$
\begin{aligned}
P(\omega, k)= & \lim _{T \rightarrow \infty} \frac{1}{T L} \\
L & \rightarrow \infty \\
& \times\left|\int_{-(T / 2)}^{T / 2} \int_{-(L / 2)}^{L / 2} x(t, s) e^{-i \omega t} e^{-i k s} d t d s\right|^{2},
\end{aligned}
$$

where $k$ is the wave number, $T$ the period, and $L$ the distance between two points. This quantity is particularly useful to assess the performances of a linear accelerator, as it contains all the information about the absolute and 
relative motions. For example, the normalized mutual spectrum (6) between two points separated by a distance $L$ can be expressed as [13]

$$
\gamma_{x y}(\omega)=\frac{\int_{0}^{\infty} P(\omega, k) \cos (k L) d k}{\int_{0}^{\infty} P(\omega, k) d k} .
$$

Using a corrected version of the well-known ATL law [3], models of the ground motion have been developed in [5] and validated on experimental measurements, assuming that the ground motion is spatially homogenous. The general expression is given by

$$
P(\omega, k)=\frac{A}{\omega^{2} k^{2}}\left[1-\cos \left(L_{0} k\right)\right]+D(\omega) U(\omega, k),
$$

where

$$
\begin{aligned}
U(\omega, k) & =\frac{2}{\sqrt{k_{\max }^{2}-k^{2}}} \quad \text { if }|k| \leq k_{\max } \\
U(\omega, k) & =0 \quad \text { if }|k|>k_{\max } \\
D(\omega) & =\frac{a_{i}}{1+\left[d_{i}\left(\omega-\omega_{i}\right) / \omega_{i}\right]^{4}}
\end{aligned}
$$

and $L_{0}=B /\left(A \omega^{2}\right) . A, B, a_{i}, d_{i}$, and $v_{i}$ are the parameters of the model; $i=1, \ldots, n$, where $n$ is the number of waves propagating in the ground. Several sets of these parameters have been calibrated in [5] in order to develop a model of the ground motion along a straight line in various locations. One of them has been developed to represent the ground motion in the LHC tunnel. It will be compared to the new measurements in the next section.

\section{GROUND VIBRATION MEASUREMENTS IN THE LHC TUNNEL}

A series of new measurements have been performed in the LHC tunnel in 2008 just before the first beams [14]. The exact location is shown in Fig. 2. The campaign has

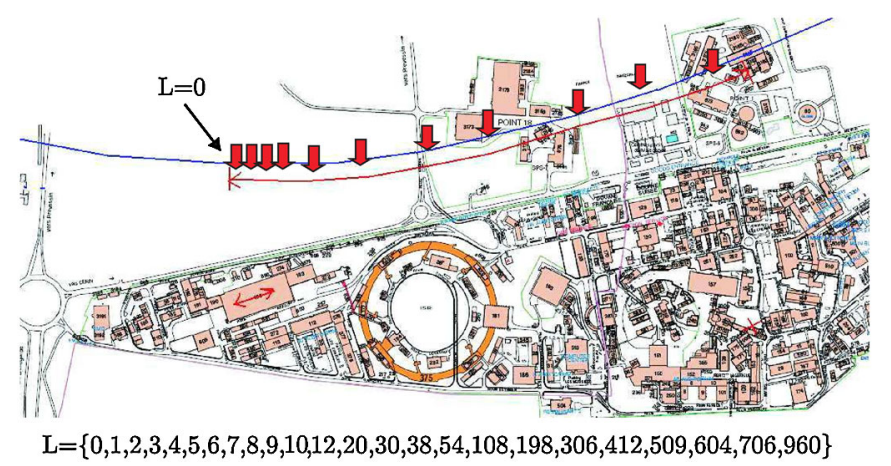

FIG. 2. (Color) Location of the ground vibration measurements in the LHC tunnel (average depth: $80 \mathrm{~m}$ ). Vertical arrows show some of the points where the signal has been recorded. been conducted as follows. The motion of the ground has been recorded by two geophones, placed at a variable distance $L$ from each other. One geophone remains always at the same place; the other one is displaced at various points in the tunnel. Some of these points are mentioned by the vertical arrows in Fig. 2. All the data have been recorded during three consecutive nights, between 11:00 PM and 4:00 AM.

Several studies of the ground motion in the LHC tunnel are reported in the literature (see e.g. [15-19]). The difference between former measurements and those presented in this paper is that they have a certain number of specific features. First, for all the distances separating the geophones, the signals have been recorded synchronously, using optical fibers. Second, the signals have been recorded in the three directions. Third, they have been recorded in accelerator operating conditions. For all the measurements, the coherence between the signals from the two geophones can be computed using Eq. (6). The result is shown in Fig. 3 for the three directions, for a few selected values of $L$.

From this figure, one sees that the motion is coherent over a long distance only in the narrow frequency range around the frequency of the microseismic wave, at $0.17(\mathrm{~Hz})$. Above that frequency, the coherence is fading out rapidly. Further evidence of the microseismic wave is given in Fig. 4, showing the velocities of two points
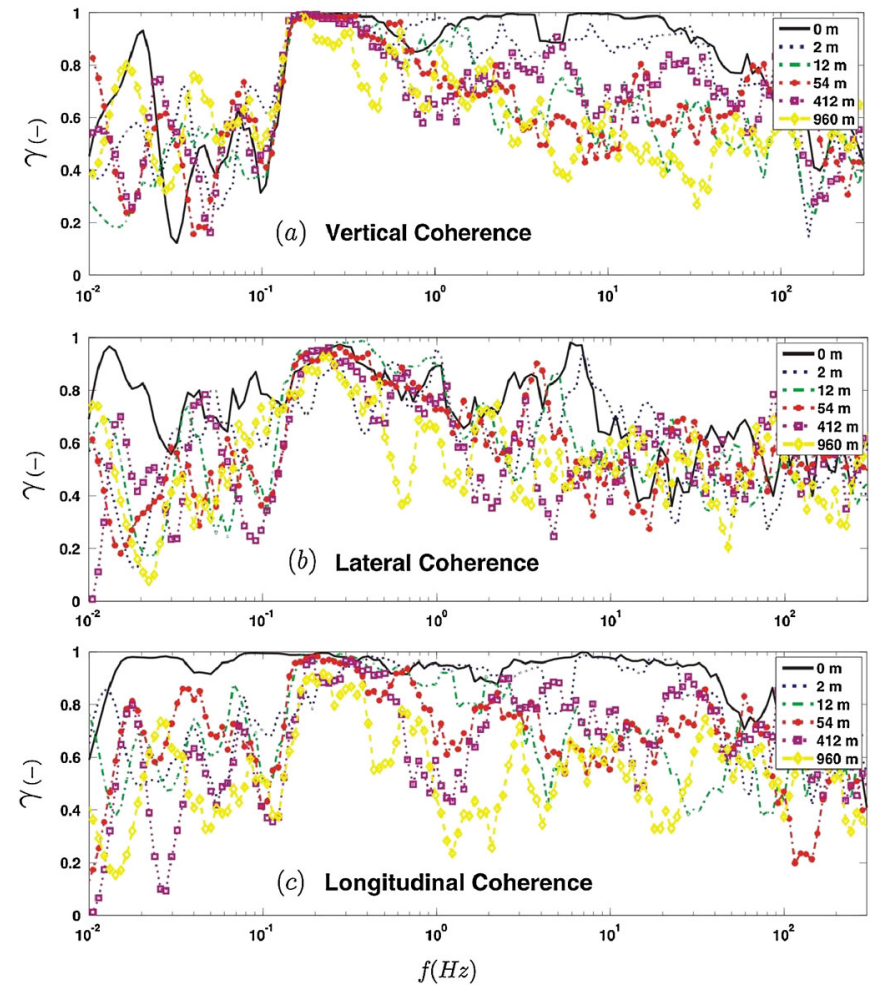

FIG. 3. (Color) Coherence measured in the three directions: (a) vertical, (b) lateral, and (c) longitudinal (i.e. along the main line of the tunnel). 

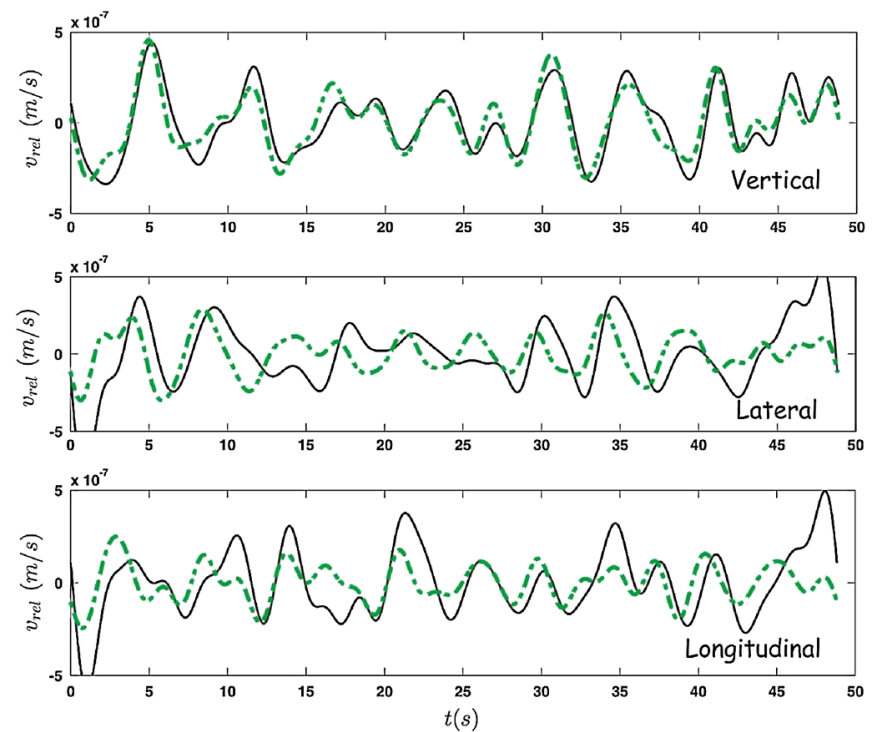

FIG. 4. (Color) Velocities of two points located at a distance of $960 \mathrm{~m}$, filtered between 0.08 and $0.5 \mathrm{~Hz}$, in the three directions.

separated by a distance of $960(\mathrm{~m})$, filtered between 0.08 and $0.5(\mathrm{~Hz})$.

Figure 5(a) shows the power spectral density $\Phi_{x}(\omega)$ of the vertical displacement at various places in the LHC tunnel. Below $1 \mathrm{~Hz}$, all the curves are superimposed. Above that, the power spectral density varies by a factor up to 10000 , i.e., the level of the signal varies by a factor 100. These sources are responsible of what is known as the technical noise. They can have two origins: either a variation of the level of vibration as a function of the abscissa of the tunnel, due to local excitation sources (e.g. cooling systems or ventilation), or a variation of the amplitude corresponding to the different times of measurements. In the latter case, the variations should also be visible on the signals from the geophone which stays always at the same location. Such variations have not been noticed. A detailed analysis of these local sources would require a synchronous monitoring of the ground vibrations at all these points in the tunnel, during several nights. This is hardly realizable, and anyway not currently feasible because the LHC machine is running. However, our experience is that the level of ground vibrations, measured at the same place and roughly the same time shows, under the same environmental conditions, roughly the same signals. For this reason, the most probable origin of these high variations of the technical noise is a variation of the level as a function of the abscissa of the tunnel.

Similarly, Fig. 6(a) shows the power spectral density $\Phi(\omega)$ of the lateral displacement at various places in the LHC tunnel. The figure also shows significant variations of the power spectral density above $1(\mathrm{~Hz})$. The procedure to take these local variations into account is explained in the next section.
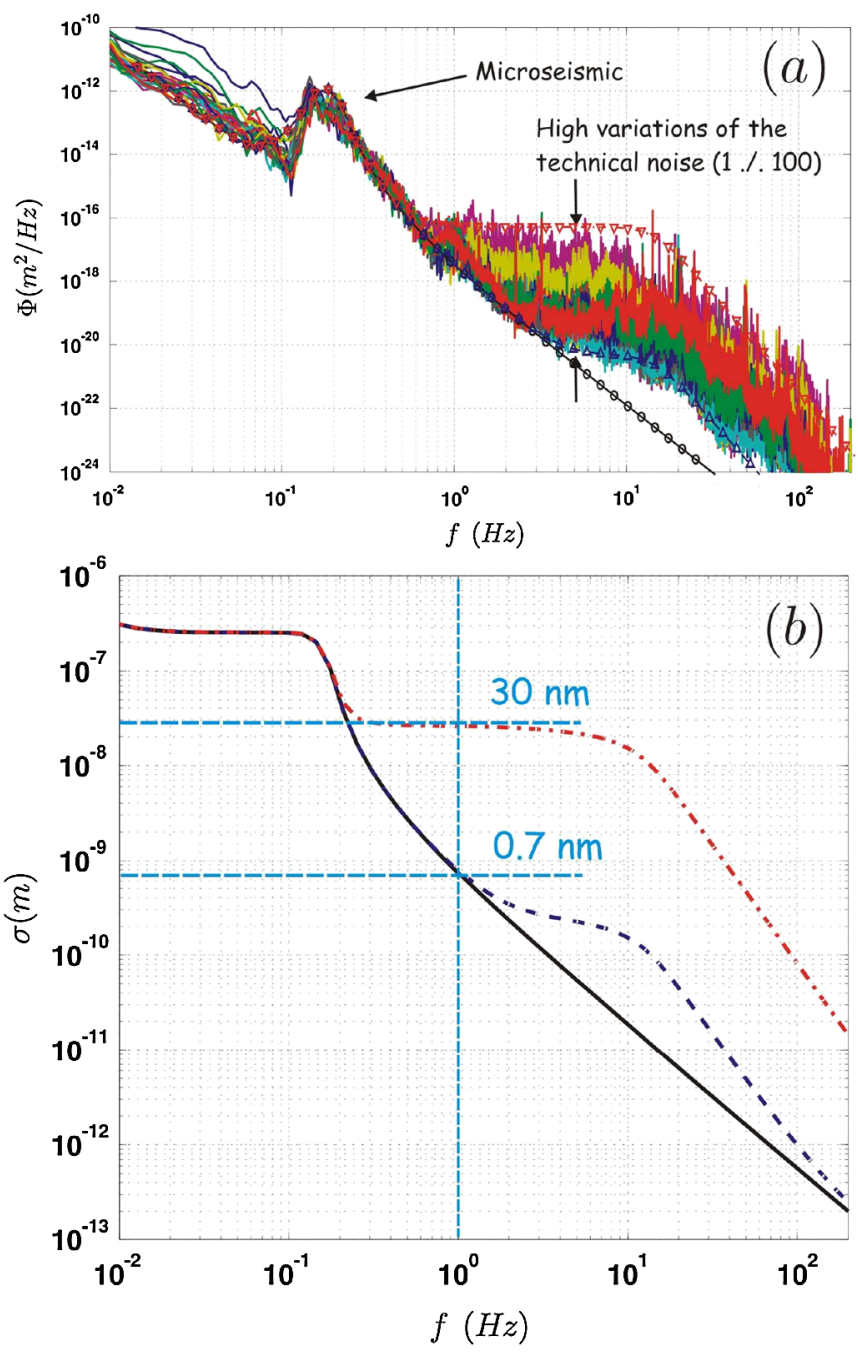

FIG. 5. (Color) (a) Power spectral densities of the measured vertical displacement in the LHC tunnel; model extracted from the measurements (solid circled line); lower and upper envelopes of the measured PSDs. (b) Integrated RMS displacements of the model (solid line) and the lower (dashed line) and upper (dashdotted line) envelopes shown in (a).

\section{UPDATED MODEL OF THE GROUND MOTION IN THE LHC TUNNEL}

Consider the lattice of quadrupoles represented in Fig. 7 , fixed on independent girders. Let $\ddot{\mathbf{u}}(t)=\left\{\ddot{u}_{1}(t), \ddot{u}_{2}(t), \ldots\right.$, $\left.\ddot{u}_{n}(t)\right\}$ be the vector of excitations at the supports of the girders.

The locations of the supports are represented in Fig. 8(a) as a function of the abscissa of the tunnel. We would like to have a quantity that could be used to represent both the absolute spectral density $\Phi_{u_{i} u_{i}}(\omega)$ at each of these supports, as well as the cross-spectral density $\rho\left(\omega, u_{i}, u_{j}\right)$ between support $i$ and support $j$ for $\{i, j\}=\{1, \ldots, n\}$. This is represented by Fig. 8(c). In practice however, only a few cross-spectral densities are measured (see Sec. III). These are represented in Fig. 8(b). 

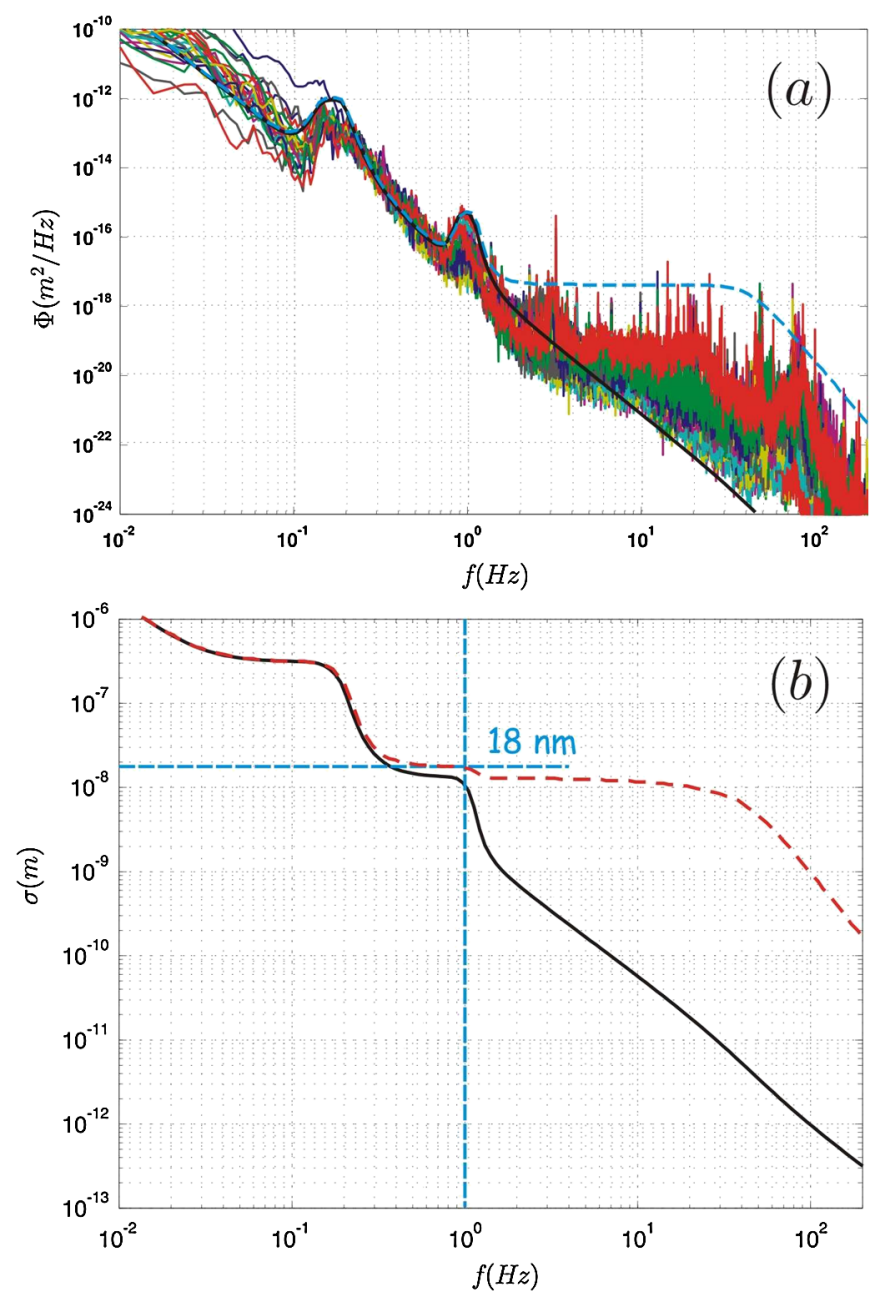

FIG. 6. (Color) (a) Power spectral densities of the measured lateral displacement in the LHC tunnel; model extracted from the measurements (solid line) and upper envelope of the PSDs (dashed line). (b) Integrated RMS displacements for the model (solid line) and the upper envelope (dashed line) shown in (a).

First of all, let us assume that the ground excitation is stationary and homogenous, i.e. $\rho\left(\omega, u_{i}, u_{j}\right)=\rho\left(\omega, u_{i}-\right.$ $u_{j}$ ), and calculate the 2D spectral density (10). In this case, the spatial variability of the ground motion is characterized by $\rho\left(\omega, L_{i}\right)$, for $i=1, \ldots, n$. Let us denote the few relative spectra available from the measurements by $\rho\left(\omega, L_{j}^{\prime}\right)$, with

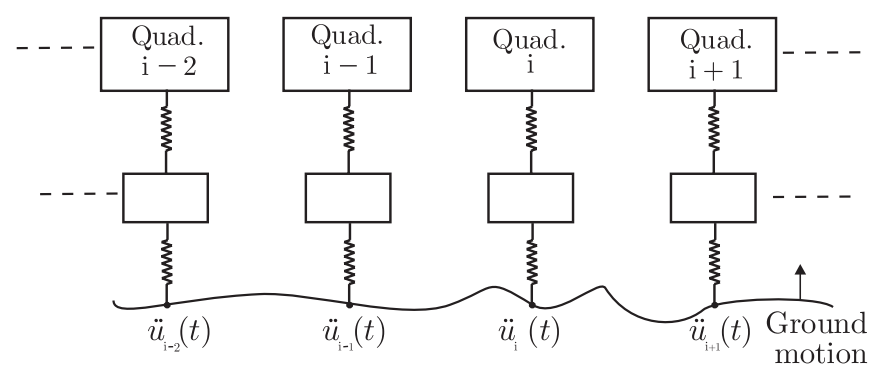

FIG. 7. General layout of the main line of a linear accelerator.

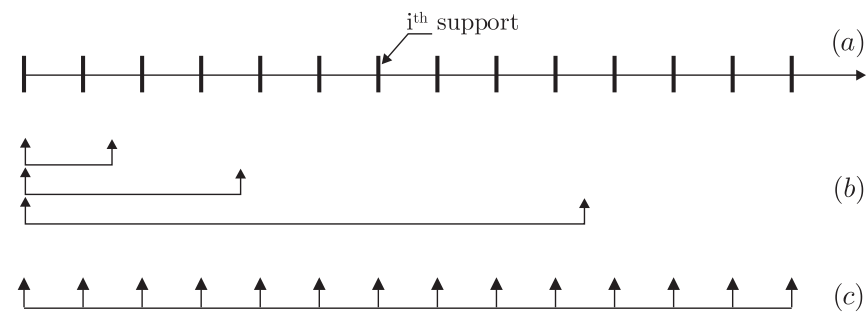

FIG. 8. (a) Locations of the supports on the longitudinal axis of the tunnel. (b) Locations of synchronous measurements of the ground motion. (c) Full characterization of the ground motion.

$j=1, \ldots, m \ll n$. Then, the relative spectra are simply related to the two-dimensional spectrum by the formula $[5,13]$

$$
P(\omega, k)=\int_{0}^{\infty} \cos (k L)[\rho(\omega, L \rightarrow \infty)-\rho(\omega, L)] d L .
$$

As the relative spectrum is available for only $m$ values, Eq. (13) becomes

$$
P(\omega, k)=\sum_{j=1}^{m} \cos \left(k L_{j}^{\prime}\right)\left[\rho\left(\omega, L_{m}\right)-\rho\left(\omega, L_{j}^{\prime}\right)\right] \Delta L .
$$

Then, the relative spectrum for any other distance $L_{i}$ is calculated from the dual equation

$$
\rho\left(\omega, L_{i}\right)=\int_{-\infty}^{+\infty} P(\omega, k) 2\left[1-\cos \left(k L_{i}\right)\right] \frac{d k}{2 \pi} .
$$

However, this method cannot give any satisfactory result because it averages all the local variations of the ground motion in the calculation of the $2 \mathrm{D}$ spectral density. The technical noise will then be overestimated when it is low, and underestimated when it is high. For this reason another method has been introduced in [20], based on asymptotic behaviors. Following this method, the following parameters have been found to model the vertical ground motion in the LHC tunnel model with Eq. (12): $A=$ $10^{-4}\left(\mu \mathrm{m}^{2} \mathrm{~s}^{-1} \mathrm{~m}^{-1}\right) ; \quad B=10^{-4}\left(\mu \mathrm{m}^{2} \mathrm{~s}^{-3}\right) ; \quad \omega_{1}=$ $2 \pi \times 0.14(\mathrm{rad} / \mathrm{s}) ; \quad d_{1}=5 ; \quad a_{1}=0.1\left(\mu \mathrm{m}^{2} / \mathrm{Hz}\right) ; \quad v_{1}=$ $1000(\mathrm{~m} / \mathrm{s})$. The coefficients are slightly different from those presented in [5]. Integrating over the wave number gives the power spectral density represented by the black solid line in Fig. 5(a). One sees on this figure that the model fits well the experimental data up to $0.8(\mathrm{~Hz})$, but does not include the technical noise. Further evidence of this difference above $0.8(\mathrm{~Hz})$ is shown in Fig. 9. Figure 9(a) shows the coherence calculated using Eq. (11) and the new numerical values for the parameters of $P(\omega, k)$. Figure 9(b) shows the coherence directly calculated from the measurements and Eq. (6).

In order to take the variations of the technical noise into account, the spectral density can be corrected by an additional analytical function like 


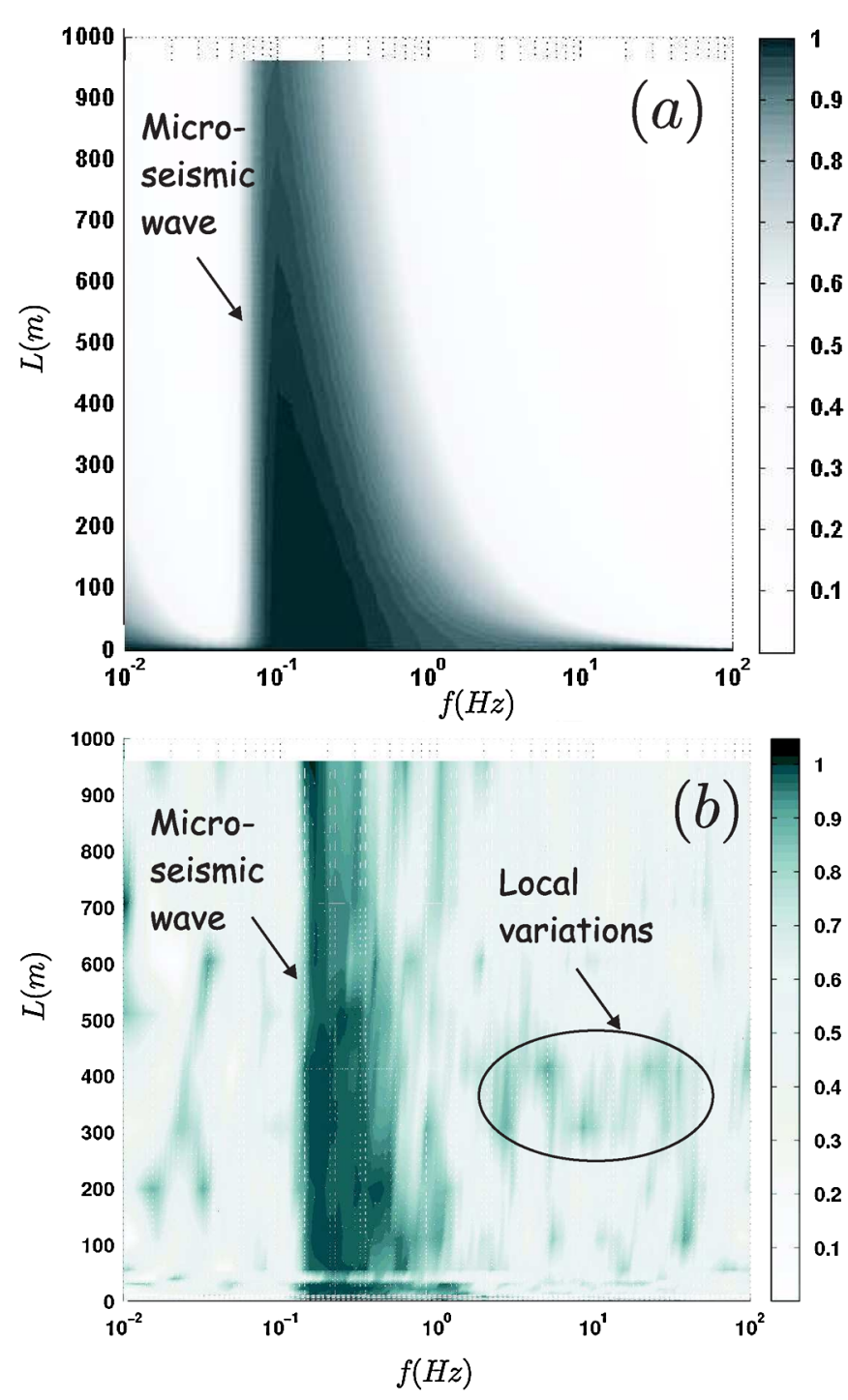

FIG. 9. (Color) Coherence between two points as a function of the distance between these points (a) for the model extracted from the measurements, and Eq. (11); (b) directly calculated from the measurements in the LHC tunnel with Eq. (6).

$$
N(\omega)=\frac{N_{0}}{1+\left(\frac{\omega}{\omega_{0}}\right)^{6}},
$$

where $\quad \omega_{0}=2 \pi f_{0} \quad$ and $\quad f_{0}=2 \pi(\mathrm{Hz}) . \quad N_{0}=5 \times$ $10^{-3}\left(\mathrm{~nm}^{2} / \mathrm{Hz}\right)$ for low technical noise and $N_{0}=$ $50\left(\mathrm{~nm}^{2} / \mathrm{Hz}\right)$ for high technical noise. These functions correspond to the upper and lower envelopes of the power spectral densities [Fig. 5(a)]. Figure 5(b) shows the integrated RMS displacements of the model without (solid line) and with the low (dashed line) and high (dash-dotted line) technical noise corrections. In the case of high technical noise, the correction leads to an integrated RMS at $1(\mathrm{~Hz})$ which is more than 40 times higher than the value predicted by the model without technical noise. This means that, in order to have a more realistic representation of the seismic excitation of a linear accelerator, these corrections should be included at several locations where the technical noise is thought to be high (e.g. where there will be a pump or a ventilation system).

Similarly, a model for the lateral direction has been obtained with the following parameters: $A=$ $10^{-3}\left(\mu \mathrm{m}^{2} \mathrm{~s}^{-1} \mathrm{~m}^{-1}\right) ; B=10^{-2}\left(\mu \mathrm{m}^{2} \mathrm{~s}^{-3}\right) ; \omega_{1}=2 \pi \times$ $0.17(\mathrm{rad} / \mathrm{s}) ; \quad d_{1}=5 ; \quad a_{1}=0.5\left(\mu \mathrm{m}^{2} / \mathrm{Hz}\right) ; \quad v_{1}=$ $1000(\mathrm{~m} / \mathrm{s}) ; \quad \omega_{2}=2 \pi \times 1(\mathrm{rad} / \mathrm{s}) ; \quad d_{2}=8 ; \quad a_{2}=$ $5 \times 10^{-4}\left(\mu \mathrm{m}^{2} / \mathrm{Hz}\right) ; v_{2}=400(\mathrm{~m} / \mathrm{s})$. Again, the local increase of the technical noise can be taken into account using Eq. (16) with $f_{0}=40(\mathrm{~Hz})$ and $N_{0}=0.5\left(\mathrm{~nm}^{2} / \mathrm{Hz}\right)$. The model without and with the correction for the technical noise are both shown in Fig. 6(a). Figure 6(b) shows the RMS integrated of the updated model, with (dashed lined) and without (solid line) the correction.

The next section presents a way to include the local excitations and accelerator dynamics in the calculation of the seismic response of a linear accelerator.

\section{DYNAMIC RESPONSE OF A LINEAR ACCELERATOR TO SPATIALLY VARYING GROUND MOTION}

An easy way to evaluate the mean square value of the change of the beam position at the interaction point is to use directly the two-dimensional power spectral density [13]

$$
\sigma_{\Delta}^{2}=\int_{-\infty}^{\infty} \int_{-\infty}^{\infty} P(\omega, k) 2\left[1-\cos \left(\omega T_{\text {rep }}\right)\right] G(k) \frac{d \omega}{2 \pi} \frac{d k}{2 \pi},
$$

where $T_{\text {rep }}$ is the repetition period of the collider, and $G(k)$ is a function of the collider parameters. Even if, due to its compactness, this relationship is very convenient for a first evaluation of the accelerator performance, it is not straightforward to include local support dynamics (e.g. the future linear accelerator CLIC will contain four different types of modules) and local variations of the ground motion (see [21] and Sec. IV).

Another approach is to take the inverse Fourier transform of the ground motion, and perform the simulation in the time domain. In this case, the displacement of the ground is given as a function of the abscissa $s$ of the tunnel by

$$
\begin{aligned}
x(t, s)= & \sum_{i} \sum_{j} a_{i j}\left\{\sin \left(\omega_{i} t\right) \sin \left(k_{j} s+\phi_{i j}\right)\right. \\
& \left.+\left[\cos \left(\omega_{i} t\right)-1\right] \sin \left(k_{j} s+\psi_{i j}\right)\right\},
\end{aligned}
$$

where $\phi_{i j}$ and $\psi_{i j}$ are random phases. The main advantage of this approach is that it can simulate transient dynamics, and nonlinear effects. However, the dynamics of the supports are usually not taken into account, as integrating the dynamic equations of a $48(\mathrm{~km})$ long machine in the time domain is time consuming. 
The approach developed in this section is to consider the accelerator as a multisupported structure [4], and solve the dynamic equations in the frequency domain. Consider a lattice constituted by a set of girders on the top of which are mounted quadrupoles (Fig. 8). The structure is excited by the motion of its supports $\mathbf{u}(t)$. Partitioning the restrained and the unrestrained degrees of freedom (d.o.f.), the dynamics of the system reads

$$
\begin{gathered}
\left(\begin{array}{ll}
\mathbf{M}_{l l} & \mathbf{M}_{l g} \\
\mathbf{M}_{g l} & \mathbf{M}_{g g}
\end{array}\right)\left(\begin{array}{c}
\ddot{\mathbf{x}} \\
\ddot{\mathbf{u}}
\end{array}\right)+\left(\begin{array}{ll}
\mathbf{C}_{l l} & \mathbf{C}_{l g} \\
\mathbf{C}_{g l} & \mathbf{C}_{g g}
\end{array}\right)\left(\begin{array}{c}
\dot{\mathbf{x}} \\
\dot{\mathbf{u}}
\end{array}\right) \\
+\left(\begin{array}{ll}
\mathbf{K}_{l l} & \mathbf{K}_{l g} \\
\mathbf{K}_{g l} & \mathbf{K}_{g g}
\end{array}\right)\left(\begin{array}{l}
\mathbf{x} \\
\mathbf{u}
\end{array}\right)=\left(\begin{array}{c}
0 \\
\mathbf{f}_{g}
\end{array}\right),
\end{gathered}
$$

where subscript $l$ refers to the lattice d.o.f. and subscript $g$ refers to those of the supports; $\mathbf{f}_{g}$ represents the excitation force at the supports, that is, the support reactions. The part of Eq. (19) relative to the unrestrained d.o.f. can be rewritten

$$
\mathbf{M}_{l l} \ddot{\mathbf{x}}+\mathbf{C}_{l l} \dot{\mathbf{x}}+\mathbf{K}_{l l} \mathbf{x}=-\mathbf{M}_{l g} \ddot{\mathbf{u}}-\mathbf{C}_{l g} \dot{\mathbf{u}}-\mathbf{K}_{l g} \mathbf{u}
$$

The response of the structure can be split into its quasistatic (qs) and dynamic components,

$$
\mathbf{x}=\mathbf{x}^{d}+\mathbf{x}^{\mathrm{qs}},
$$

where $\mathbf{x}^{\mathrm{qs}}$ stands for the quasistatic response of the structure resulting from the support displacements, and $\mathbf{x}^{d}$ the dynamic response. $\mathbf{x}^{\mathrm{qs}}$ can be found by canceling the time derivatives in Eq. (20):

$$
\mathbf{x}^{\mathrm{qs}}=-\mathbf{K}_{l l}^{-1} \mathbf{K}_{l g} \mathbf{u} .
$$

Substituting Eq. (21) into Eq. (20) and using Eq. (22) gives

$$
\begin{aligned}
\mathbf{M}_{l l} \ddot{\mathbf{x}}^{d}+\mathbf{C}_{l l} \dot{\mathbf{x}}^{d}+\mathbf{K}_{l l} \mathbf{x}^{d}= & \left(\mathbf{M}_{l l} \mathbf{K}_{l l}^{-1} \mathbf{K}_{l g}-\mathbf{M}_{l g}\right) \ddot{\mathbf{u}} \\
& +\left(\mathbf{C}_{l l} \mathbf{K}_{l l}^{-1} \mathbf{K}_{l g}-\mathbf{C}_{l g}\right) \dot{\mathbf{u}} .
\end{aligned}
$$

Assuming that the damping matrix is proportional to the stiffness matrix, the last term of (23) vanishes and one gets

$$
\mathbf{M}_{l l} \ddot{\mathbf{x}}^{d}+\mathbf{C}_{l l} \dot{\mathbf{x}}^{d}+\mathbf{K}_{l l} \mathbf{x}^{d}=\left(\mathbf{M}_{l l} \mathbf{K}_{l l}^{-1} \mathbf{K}_{l g}-\mathbf{M}_{l g}\right) \ddot{\mathbf{u}} .
$$

In the frequency domain, the dynamic part of the response is obtained by taking the Fourier transform of Eq. (24),

$$
\mathbf{X}^{d}(\omega)=\mathbf{G}(\omega)\left[\left(\mathbf{M}_{l l} \mathbf{K}_{l l}^{-1} \mathbf{K}_{l g}-\mathbf{M}_{l g}\right)\right] \ddot{\mathbf{U}}(\omega),
$$

where $\mathbf{G}(\omega)=\left[-\omega^{2} \mathbf{M}_{l l}+j \omega \mathbf{C}_{l l}+\mathbf{K}_{l l}\right]^{-1}$. Similarly, taking the Fourier transform of Eq. (22), one gets

$$
\mathbf{X}^{q s}(\omega)=-\mathbf{K}_{l l}^{-1} \mathbf{K}_{l g} \mathbf{U}(\omega)=\frac{-\mathbf{K}_{l l}^{-1} \mathbf{K}_{l g}}{\omega^{2}} \ddot{\mathbf{U}}(\omega) .
$$

Combining Eqs. (19), (21), and (22), the total response of the system is given by

$$
\mathbf{X}(\omega)=\left[\frac{\mathbf{A}}{\omega^{2}}+\mathbf{G}(\omega) \boldsymbol{\Gamma}\right] \ddot{\mathbf{U}}(\omega),
$$

where $\mathbf{A}=-\mathbf{K}_{l l}^{-1} \mathbf{K}_{l g}$ and

$$
\boldsymbol{\Gamma}=\mathbf{M}_{l l} \mathbf{K}_{l l}^{-1} \mathbf{K}_{l g}-\mathbf{M}_{l g}
$$

is called the participation matrix.

As the response of the structure is dominated by a few modes, it can be more convenient to decompose the dynamic displacements into their modal components $\mathbf{z}$ according to

$$
\mathbf{x}^{d}=\mathbf{\Phi} \mathbf{z}
$$

where $\boldsymbol{\Phi}=\left\{\phi_{1}, \phi_{2}, \ldots, \phi_{m}\right\}$ is a $n \times m$ matrix ( $n$ is the number of d.o.f. and $m$ is the number of modes considered in the study) whose columns are the normal modes of the fixed base structure, satisfying the orthogonality conditions

$$
\begin{aligned}
& \boldsymbol{\Phi}^{T} \mathbf{M}_{l l} \phi=\mu=\operatorname{diag}\left(\mu_{i}\right) \\
& \boldsymbol{\Phi}^{T} \mathbf{K}_{l l} \boldsymbol{\Phi}=\mu \boldsymbol{\Omega}^{2}=\operatorname{diag}\left(\mu_{i} \omega_{i}^{2}\right),
\end{aligned}
$$

where $\mu_{i}$ and $\omega_{i}$ are, respectively, the mass and frequency of mode $i$. Assuming that $\boldsymbol{\Phi}^{T} \mathbf{C}_{l l} \Phi=2 \Xi \boldsymbol{\Omega}=2 \xi_{i} \omega_{i}$, Eq. (24) becomes

$$
\mu \ddot{\mathbf{z}}+2 \Xi \boldsymbol{\Omega} \dot{\mathbf{z}}+\mu \boldsymbol{\Omega}^{2}=\Gamma^{m} \ddot{\mathbf{u}},
$$

where

$$
\boldsymbol{\Gamma}^{m}=\boldsymbol{\Phi}^{T}\left(\mathbf{M}_{l l} \mathbf{K}_{l l}^{-1} \mathbf{K}_{l g}-\mathbf{M}_{l g}\right)
$$

is the $m \times n_{s}$ modal participation matrix.

Upon Fourier transforming Eq. (31), the modal amplitudes are given by

$$
\mathbf{Z}(\omega)=\mathbf{H}(\omega) \Gamma^{m} \ddot{\mathbf{U}}(\omega),
$$

where

$$
\mathbf{H}(\omega)=\frac{1}{\operatorname{diag}\left[\mu_{i}\left(\omega_{i}^{2}-\omega^{2}+2 j \xi_{i} \omega \omega_{i}\right)\right]} .
$$

Combining Eqs. (29) and (33) into (27), one gets

$$
\mathbf{X}(\omega)=\left[\frac{\mathbf{A}}{\omega^{2}}+\boldsymbol{\Phi}^{T} \mathbf{H}(\omega) \boldsymbol{\Gamma}\right] \ddot{\mathbf{U}}(\omega) .
$$

The power spectral density of the response is found by applying the definition (2) to the above equation [22-24]: 


$$
\begin{aligned}
\mathbf{S}_{X X}(\omega)= & \left\{\left[\frac{\mathbf{A}}{\omega^{2}}+\boldsymbol{\Phi}^{T} \mathbf{H}(\omega) \boldsymbol{\Gamma}\right]\right\} \mathbf{S}_{\ddot{\mathbf{u}} \ddot{\mathbf{u}}}(\omega)\left\{\left[\frac{\mathbf{A}}{\omega^{2}}+\boldsymbol{\Phi}^{T} \mathbf{H}(\omega) \boldsymbol{\Gamma}\right]\right\}^{*} \\
= & \frac{1}{\omega^{4}} \sum_{l=1}^{N} \sum_{m=1}^{N} A_{i l} A_{i m} \Phi_{\ddot{u}_{l} \ddot{u}_{m}}(\omega)+\sum_{j=1}^{n} \sum_{k=1}^{n} \sum_{l=1}^{N} \sum_{m=1}^{N} \phi_{i j} \phi_{i k} \Gamma_{l j} \Gamma_{m k} H_{j}(\omega) H_{j}^{*}(\omega) \Phi_{\ddot{u}_{l} \ddot{u}_{m}}(\omega) \\
& -\frac{1}{\omega^{2}} \sum_{j=1}^{n} \sum_{l=1}^{N} \sum_{m=1}^{N} \phi_{i j} A_{i l} \Gamma_{m j} H_{j}(\omega) \Phi_{\ddot{u}_{l} \ddot{u}_{m}}(\omega) \\
= & S_{X^{\mathrm{qs}} X^{\mathrm{qs}}}(\omega)+S_{X^{d} X^{d}}(\omega)+S_{X^{d} X^{\mathrm{qs}}}(\omega)
\end{aligned}
$$

where $n$ is the number of unrestrained d.o.f. and $N$ is the number of restrained d.o.f. The variance of Eq. (21) gives

$$
\begin{aligned}
\sigma_{X}^{2}= & \sigma_{X^{q} s}^{2}+\sigma_{X^{d}}^{2}+2 \operatorname{cov}\left(X^{d}, X^{\mathrm{qs}}\right) \\
= & \sum_{l=1}^{N} \sum_{m=1}^{N} A_{i l} A_{i m} \int_{-\infty}^{\infty} \frac{1}{\omega^{4}} \Phi_{\ddot{u}_{l} \ddot{u}_{m}}(\omega) d \omega+\sum_{j=1}^{n} \sum_{k=1}^{n} \sum_{l=1}^{N} \sum_{m=1}^{N} \phi_{i j} \phi_{i k} \Gamma_{l j} \Gamma_{m k} \int_{-\infty}^{\infty} H_{j}(\omega) H_{j}^{*}(\omega) \Phi_{\ddot{u}_{l} \ddot{u}_{m}}(\omega) d \omega \\
& -\sum_{j=1}^{n} \sum_{l=1}^{N} \sum_{m=1}^{N} \phi_{i j} A_{i l} \Gamma_{m j} \int_{-\infty}^{\infty} \frac{1}{\omega^{2}} H_{j}(\omega) \Phi_{\ddot{u}_{l} \ddot{u}_{m}}(\omega) d \omega
\end{aligned}
$$

which gives the mean square value of any d.o.f. of the structure. The integrated RMS displacement $\sigma_{X}(\omega)$ is readily found by replacing the lower integration bound by $\omega$ in Eq. (37) and taking the square root of the whole expression. The first term represents the contribution of the quasistatic response of the lattice; the second term represents the dynamic contribution; the third term represents the interaction between the quasistatic and dynamic contribution.

The excitation matrix appearing in (36) can be expanded as

$$
\mathbf{S}_{\ddot{\mathbf{u}} \ddot{\mathbf{u}}}(\omega)=\left(\begin{array}{cccc}
\Phi_{\ddot{u}_{1} \ddot{u}_{1}}(\omega) & \Phi_{\ddot{u}_{1} \ddot{u}_{2}}(\omega) & \cdots & \Phi_{\ddot{u}_{1} \ddot{u}_{n}}(\omega) \\
\Phi_{\ddot{u}_{2} \ddot{u}_{1}}(\omega) & \Phi_{\ddot{u}_{2} \ddot{u}_{2}}(\omega) & \cdots & \Phi_{\ddot{u}_{2} \ddot{u}_{n}}(\omega) \\
\cdots & \cdots & \cdots & \cdots \\
\Phi_{\ddot{u}_{n} \ddot{u}_{1}}(\omega) & \Phi_{\ddot{u}_{n} \ddot{u}_{1}}(\omega) & \cdots & \Phi_{\ddot{u}_{n} \ddot{u}_{n}}(\omega)
\end{array}\right),
$$

where $\Phi_{\ddot{i}_{i} \ddot{u}_{j}}$ is the cross power spectral density between the acceleration at the support $i$ and the support $j$.

In the particular case of a spatially homogenous excitation,

$$
\Phi_{\ddot{u}_{i} \ddot{u}_{j}}=\omega^{4} \int_{0}^{\infty} P(\omega, k) \cos (k L) d k,
$$

where $L$ is the distance between the support $i$ and the support $j$.

Then, in order to take local excitations into account, we can simply add the contribution from the local technical noise and the excitation matrix becomes

$$
\mathbf{S}_{\ddot{\mathbf{u}} \ddot{u}}(\omega)=\mathbf{S}_{\ddot{\mathbf{u}} \ddot{u}}(\omega)+\mathbf{S}_{\text {local }}(\omega),
$$

where

$$
\mathbf{S}_{\text {local }}(\omega)=\omega^{4} \operatorname{diag}\left\{N_{1}(\omega), \ldots, N_{n}(\omega)\right\}
$$

is a diagonal matrix because the technical noise is essentially uncorrelated, and $N_{i}$ is given by (16).

Finally, if $\mathbf{R}$ is the transfer matrix of the line, linking the quadrupole displacements and the change of the beam position at the interaction point $\Delta$, the power spectrum of $\Delta$ is given by

$$
\Phi_{\Delta}(\omega)=\mathbf{R S}_{X X}(\omega) \mathbf{R}^{*}
$$

The main drawback of this formulation is that it is far less compact than (17). The main advantage is that any type of local characteristic can be fairly easily taken into account, e.g., local excitation, local dynamics, or quadrupole flexibility.

In order to illustrate the model developed, let us consider a FODO lattice, constituted of $N$ quadrupoles, alternatively focusing and defocusing. In this case, the transfer matrix from the quadrupoles to the beam offset at the end of the lattice is a vector given by $[16,25,26]$

$$
R(j)=\frac{\sqrt{\beta^{*} \beta_{j}}}{F} \sin \left(\Psi_{j}\right) x_{j},
$$

where $F$ is the focal length of the quadrupoles, $x_{i}$ is the offset of quadrupole $i, \beta^{*}$ is the $\beta$ function at end of the lattice, 


$$
\beta_{j}=\frac{1+\sin (\mu / 2)(-1)^{j}}{\sin (\mu)} 2 s_{j}
$$

where $s_{j}=j l$ is the coordinate of cell $j$ along the lattice, and $\mu$ is the phase advance per cell. Even values of $j$ correspond to focusing quadrupoles, and odd values correspond to defocusing quadrupoles. $\Psi_{j}=(1 / 2)(j-N) \mu$ is the betatron phase advance.

In this example, it is assumed that all quadrupoles have the same dynamic response. Each quadrupole is mounted on a girder to perform the realignment of the elements, e.g., with a cam system. If we assume that each pair of quadrupole girder is a two d.o.f. system, the corresponding lattice is represented in Fig. 7. For each system, the typical transfer function $T_{u_{j} x_{j}}(f)$ between the ground motion $u_{j}$ and the quadrupole displacement $x_{j}$ is shown in Fig. 10 (dash-dotted line). Let us further assume that a mechanical stabilization of the quadrupoles can decrease this transmissibility between $0.4(\mathrm{~Hz})$ and $20(\mathrm{~Hz})$, as shown in Fig. 10 (solid line). Also, let us consider that the beam based correction system corrects the position of each pulse by subtracting the value measured at the previous pulse [6]. The ground motion model used in this example is the new model presented in this paper, with an additional low technical noise on each support. The following numerical values have been used in the simulation: $N=128, \mu=$ $\pi / 2, l=6(\mathrm{~m})$, and a pulse repetition rate of $f_{\text {rep }}=$ $30(\mathrm{~Hz})$. The power spectral density of the ground motion is shown in Fig. 11(a) (dashed line). Figure 11(a) also shows $\Phi_{\Delta}(\omega)$ at the end of the FODO lattice, when the mechanical stabilization is OFF (dash-dotted line) and ON (solid line). Figure 11(b) shows the integrated RMS of the power spectral densities shown in Fig. 11(a). These results

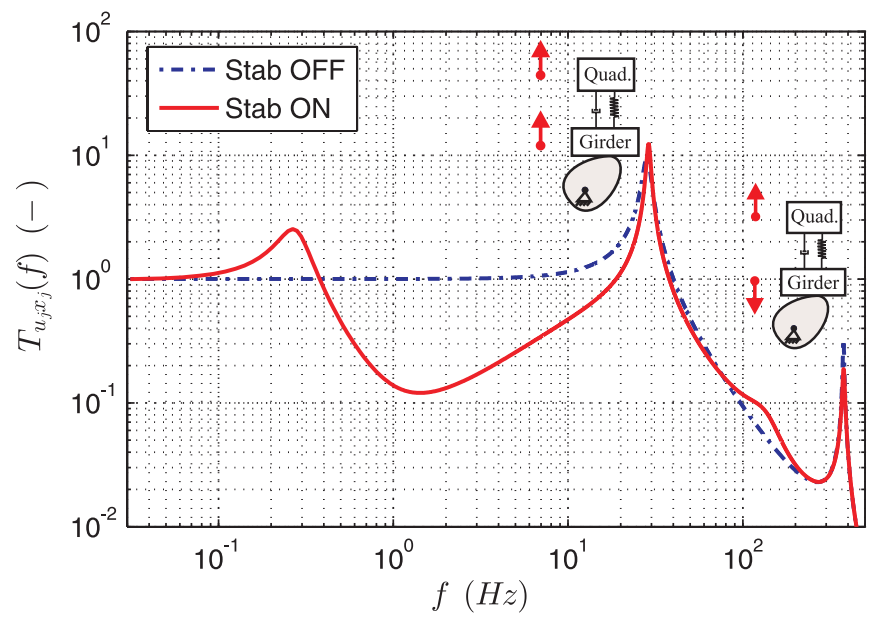

FIG. 10. (Color) Two d.o.f. model including an alignment stage and a stabilization stage. Transmissibility $T_{u_{j} x_{j}}(f)$ between the ground and the quadrupole, when the mechanical stabilization is OFF (dash-dotted line) and ON (solid line).
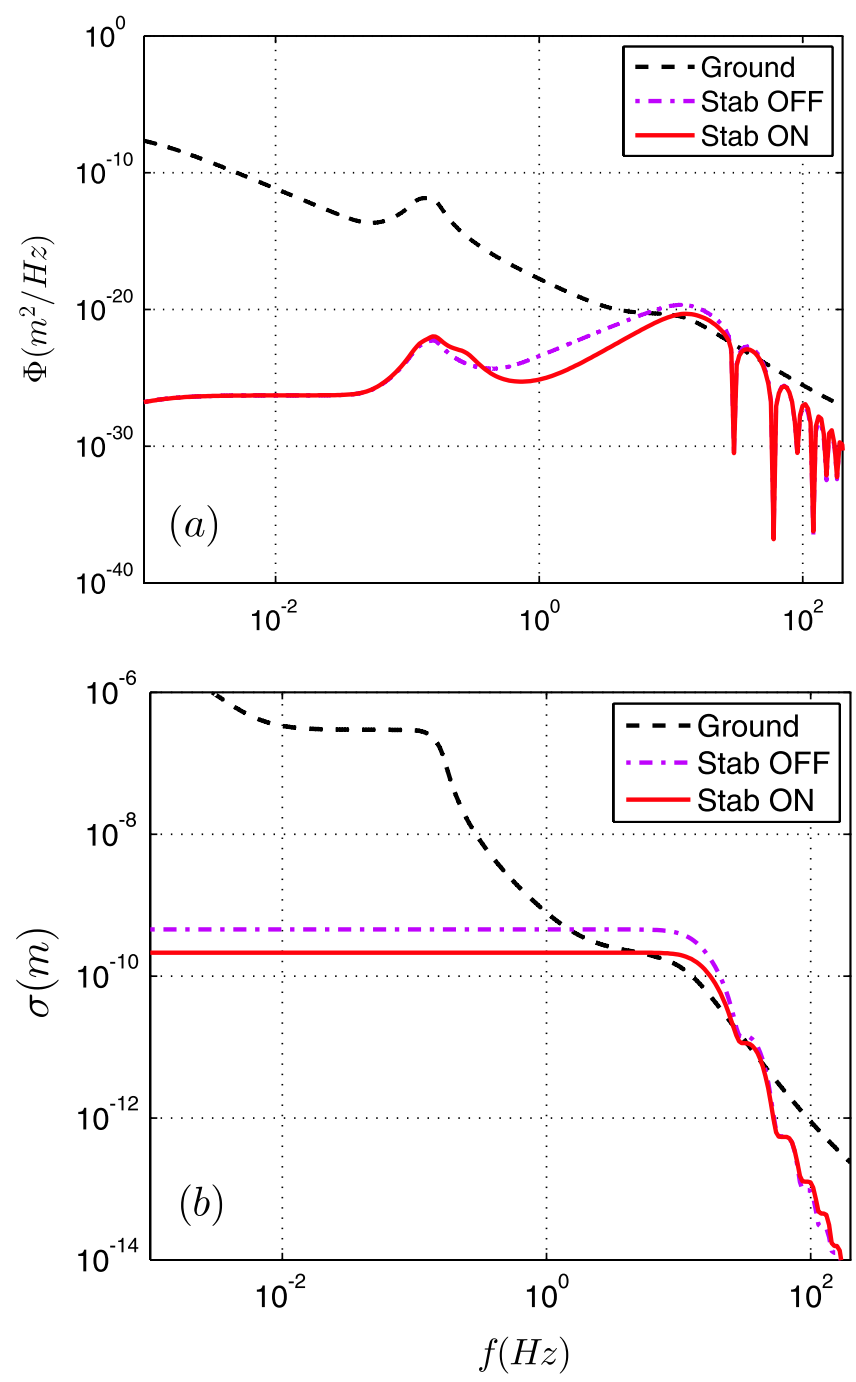

FIG. 11. (Color) (a) Power spectral density of the ground motion (dashed line), and of the beam at the end of the FODO lattice, when the mechanical stabilization is OFF (dash-dotted line) and ON (solid line). (b) Integrated RMS of the power spectral densities shown in (a).

are similar to the results presented in the literature (see e.g. [6]).

\section{CONCLUSIONS}

This paper contains mainly two contributions. First, new measurements of the ground motion in the LHC tunnel have been presented. Thanks to some of their key characteristics (synchronous and multidirectional) it has been possible to update the existing model of the ground motion in the vertical direction, and propose a similar one in the lateral direction. Also, it has been found from the measurements that the integrated RMS of the ground motion displacement can vary locally significantly. In order to have a more realistic model of the excitation in the LHC tunnel, a model of the local excitation has been presented. 
Second, a dynamic model of linear accelerator has been presented, which can include any type of local excitation and local dynamics. Specifically, it has been shown how local sources of excitations can be included in the model to evaluate the seismic response of a linear accelerator. A regular FODO lattice has been finally presented as a numerical validation of the approach, including the dynamics of the alignment stage, a mechanical stabilization of the quadrupoles, and a simple beam based correction.

\section{ACKNOWLEDGMENTS}

The research leading to these results has received funding from the European Commission under the FP7 Research Infrastructures project EuCARD, Grant Agreement No. 227579. The authors also gratefully acknowledge the reviewers for their useful comments.

[1] G. Fisher, Technical Report No. SLAC-PUB-3392-Rev, 1985.

[2] G. Fisher and M. Mayond, Technical Report No. CERNLEP-SU-88-07, CLIC note 61, 1988.

[3] B. Baklakov, P. Lebedev, V. Parkhomchuk, A. Sery, A. Sleptsov, and V. Shiltsev, in Proceedings of the Particle Accelerator Conference (IEEE, San Francisco, 1991).

[4] A. Preumont, Random Vibration and Spectral Analysis (Kluwer Academic Publisher, Dordrecht, 1994).

[5] A. Sery and O. Napoly, Phys. Rev. E 53, 5323 (1996).

[6] C. Montag, Ph.D. thesis, Hamburg University, 1996.

[7] K. Hasselmann, Rev. Geophys. 1, 177 (1963).

[8] R. Haubrich and K. McCamy, Rev. Geophys. 7, 539 (1969).

[9] G. Fisher and M. Morton, Technical Report No. SLACPUB-3870, 1986.

[10] V. Parkhomchuk, V. Shiltsev, and G. Stupakov, Technical Report No. SSCL-Preprint-470, 1993.
[11] V. Shiltsev, in 4th International Workshop on Accelerator Alignment (IWAA-93), Tsukuba, Japan, 1995.

[12] P. Lebedev, C. Montag, V. Shiltsev, B. Baklakov, and J. Rossbach, Technical Report No. DESY HERA-9506, 1995.

[13] V. Juravlev, P. Lunev, A. Sery, and A. Sleptsov, Technical Report No. HU-SEFT R 1995-01, 1995.

[14] K. Artoos, O. Capatina, C. Collette, M. Guinchard, C. Hauviller, M. Sylte, B. Bolzon, and A. Jeremie, in Proceedings of the 23rd Particle Accelerator Conference, Vancouver, Canada, 2009 (IEEE, Piscataway, NJ, 2009).

[15] V. Juravlev, A. Sery, A. Sleptsov, W. Coosemans, G. Ramseier, and I. Wilson, Technical Report No. CERNSL/93-53, CLIC note 217, 1993.

[16] A. Sery and A. Mosnier, Phys. Rev. E 56, 3558 (1997).

[17] B. Baklakov, T. Bolshakov, A. Chupyra, A. Erokhin, P. Lebedev, V. Parkhomchuk, Sh. Singatulin, J. Lach, and V. Shiltsev, Phys. Rev. ST Accel. Beams 1, 031001 (1998).

[18] A. Sery, A. Sleptsov, Yu. Valiaev, W. Coosemans, G. Ramseier, I. Wilson, V. Lawson-Chroco, V. Balakin, and V. Juravlev, Technical Report No. CERN-SL-93-30-NPL, CLIC note 191, 1993.

[19] L. Vos, Part. Accel. 50, 221 (1995).

[20] NLC ZDR Design Group, Technical report, SLAC Report No. 474, 1996.

[21] A. Sery, M. Breidenbach, and J. Frisch, in Proceedings of the 20th International Linac Conference, Monterey, CA, USA [SLAC Report No. SLAC-PUB-8594 (2000)].

[22] F. Tubino, L. Carassale, and G. Solari, Earthquake Eng. Struct. Dyn. 32, 1639 (2003).

[23] S. Ates, A. Bayraktar, and A. Dumanglu, Soil Dyn. Earthquake Eng. 26, 31 (2006).

[24] Y. Bilici, A. Bayraktar, K. Soyluk, K. Haciefendioglu, S. Ates, and S. Adnur, Soil Dyn. Earthquake Eng. 29, 444 (2009).

[25] E. Courant and H. Snyder, Ann. Phys. (N.Y.) 3, 1 (1958).

[26] G. Stupakov, Technical Report No. SLAC-PUB-8694, 1985. 